\title{
COMBINED PREIMPLANTATION GENETIC TESTING FOR ANEUPLOIDY AND MONOGENIC DISEASE IN A MEXICAN FAMILY AFFECTED BY X-LINKED HYPOHIDROTIC ECTODERMAL DYSPLASIA
}

\author{
Raul Eduardo Piña-Aguilar, Claudia González-Ortega, Anna Calull-Bago, María Cristina \\ lanuza-López, Patricia Cancino-Villarreal, Ana Mireya Gutiérrez-Gamiño and Antonio Martin \\ GutiérREZ-GutiÉRREZ*
}

Institute of Sciences in Human Reproduction “Vida”, León, Gto., Mexico

\begin{abstract}
Background: Hypohidrotic ectodermal dysplasia (HED) is a genetic skin condition presenting as hypohidrosis, hypodontia, and hypotrichosis, resulting in an important burden for affected families. The most common form of HED has an X-linked inheritance and female carriers have the option of prenatal or preimplantation genetic testing (PGT) to avoid transmission of the disease. A combined PGT for a mutation in EDA gene and aneuploidies in a Mexican carrier of X-linked HED is reported. Materials and Methods: Ovarian stimulation and assisted reproduction procedures were performed in a private academic medical center. PGT for a novel c.707-1G>A (rs886039466) mutation in EDA gene and chromosomal aneuploidies was performed by massive parallel and Sanger sequencing. Results: In the first PGT, the transfer of two blastocysts did not result in a pregnancy. An accumulative stimulation approach was decided to improve pregnancy chances for a second PGT procedure. Three ovarian stimulations were performed and 10 blastocysts coming from fresh and vitrified oocytes were genetically analyzed. A single embryo transfer produced a healthy non-carrier euploid girl. Discussion: PGT combining aneuploidy and mutation analyses is an alternative for female carriers of X-linked and other Mendelian disorders in Latin-American countries. In the era of genomic and personalized medicine, medically assisted reproduction techniques, such as PGT, are shifting from only infertility to preventive genetics. (REV INVES CLIN. 2018;70:164-8)
\end{abstract}

Key words: Christ-Siemens-Touraine syndrome. X-linked disorder. Genodermatosis. Preimplantation genetic diagnosis.

Corresponding author:

*Antonio M. Gutiérrez-Gutiérrez

Plaza Las Américas 115

Paseo del Moral y Av. Guanajuato

Col. Jardines del Moral

Received for publication: 13-04-2018

C.P. 37160, León, Gto., México

E-mail: antoniogtz@usa.net

Accepted for publication: 25-05-2018

doi: $10.24875 / R I C .18002562$ 


\section{INTRODUCTION}

Hypohidrotic ectodermal dysplasia (HED) is one of the ectodermal dysplasias, a group of approximately 200 genetic skin disorders affecting the structure of ectodermal tissues, skin, hair, nails, sweat glands, and teeth. HED is characterized by a triad of signs: hypotrichosis, anodontia or hypodontia, and anhidrosis or hypohidrosis ${ }^{1}$.

HED is caused by mutations in EDA, EDAR, EDARADD, or WNTIOA genes and inherited in an autosomal dominant, autosomal recessive, or $\mathrm{X}$-linked manner depending on the gene involved ${ }^{1}$. The majority of individuals with HED has the X-linked form (OMIM 305100) with pathogenic variants in EDA gene located at Xq13.1 and encoding for ectodysplasin- $\mathrm{A}^{1}$.

Patients carriers of an X-linked, autosomal recessive, or dominant genetic disorder have two main options to prevent the transmission of disease: prenatal diagnosis or preimplantation diagnosis. Prenatal diagnosis can be performed determining only sex (by karyotyping) or with direct mutation analysis in fetal tissue obtained from chorionic villus sampling or amniocentesis. Preimplantation genetic testing (PGT) offers the advantage that couples do not need to interrupt an already established pregnancy. However, for PGT-M, it is required to know in advance which is the mutation and set up a specific assay for embryo testing in each affected family. There are important advances to the study of genetic make-up of embryos, and now, it is becoming possible to study aneuploidies and mutations producing Mendelian disorders at the same time $^{2}$.

In this report, we describe our experience in performing a combined preimplantation genetic testing (PGT) for aneuploidy using massive parallel sequencing and single-gene defect in EDA gene in a Mexican family affected by $X$-linked HED.

\section{MATERIALS AND METHODS}

\section{Assisted reproduction procedures}

Transvaginal ultrasound scan was performed on the patient at day 2 or 3 of her menstrual cycle to verify ovarian quiescence and perform antral follicle count ${ }^{3}$.
300 IU recombinant follicle-stimulating hormone $(\mathrm{rFSH})+150$ recombinant luteinizing hormone $(\mathrm{rLH})$ (Pergoveris, Merck Serono) and 75 IU rFSH (Gonal, Merck Serono) were initiated on day 3 until oocyte retrieval and gonadotropin-releasing hormone antagonist started when one follicle reached $14 \mathrm{~mm}$. A single s.c. injection of $250 \mu \mathrm{g}$ of recombinant human chorionic gonadotropin (rhCG) (Ovidrel, Merck Serono) was administered when one follicle reached 18 $\mathrm{mm} ; 36 \mathrm{~h}$ after the hCG injection, oocyte retrieval was performed by culdocentesis using a needle and vacuum pump with the patient under IV sedation ${ }^{3}$.

Embryology procedures were modified from our previous protocols ${ }^{4}$. In retrieved cumulus-oocyte complexes, granulosa cells were removed using hyaluronidase (InVitroCare) and mechanical pipetting for the assessment of oocyte maturity. Mature (metaphase II) oocytes were injected with spermatozoa obtained after gradient centrifugation (Pure Sperm, Nidacon) combined with a swim-up procedure. Injected oocytes were transferred to Global Total medium (LifeGlobal Group) covered with paraffin oil (LifeGlobal Group) and incubated at $37^{\circ} \mathrm{C}$ in $6.5 \% \mathrm{CO}_{2}, 5 \% \mathrm{O}_{2}$ in miniincubators (Miri, Esco). After $17 \mathrm{~h}$, fertilization was evaluated by the presence of two pronuclei and two polar bodies. Embryos were cultured in $20 \mu \mathrm{il}$ droplets of Global medium at $37^{\circ} \mathrm{C}$ in $6.5 \% \mathrm{CO}_{2}$. For embryo biopsy, laser (Octax, MTG GmbH)-assisted trophectoderm biopsy of day 5 or 6 blastocysts in HEPES-HTF medium (LifeGlobal) was performed. The biopsies of trophectoderm containing 5-10 cells were transferred to polymerase chain reaction (PCR) tubes and transported to Igenomix laboratory. Zygotes and blastocysts were vitrified using Cryotop devices (Kitazato); blastocysts were maintained frozen waiting for the genetic results. Endometrial preparation was from a natural cycle, and embryos were transferred using a Wallace catheter under ultrasound guidance. Luteal supplementation with micronized progesterone 90 mg vaginal gel (Crinone, Merck Serono) for 3 months was prescribed. Levels of hCG 14 days after embryo transfer were measured, and fetal heartbeat confirmed at 35 days after embryo transfer with obstetrical follow-up of established pregnancy.

\section{Genetic analysis}

Genetic analysis was performed by Igenomix, Spain. Microsatellite markers DXS1125, DXS1275, DXS8031, 
Figure 1. Pedigree of family. Affected members are shown by underwent molecular testing.

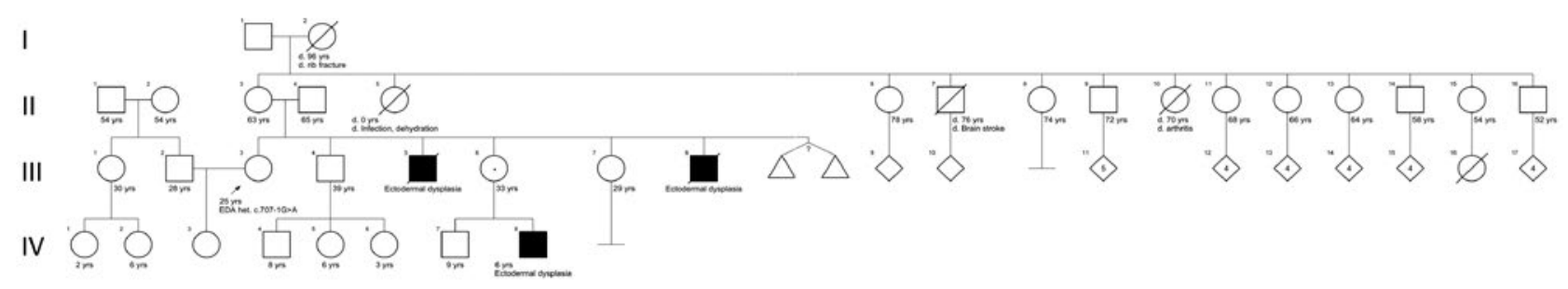

DXS1690, DXS8107, DXS8052, and the mutation c.707-1G>A were analyzed using DNA from the couple, an affected nephew, and a healthy nephew using capillary electrophoresis and minisequencing in an AB3130 machine (Thermo Fisher Scientific). This was done to confirm that PGT for the mutation was possible.

For aneuploidy screening, whole genome amplification and screening for unbalanced chromosomes were performed with Ion ReproSeq PGS kit (Thermo Fisher Scientific) using massive parallel sequencing in a PGM system and analyzed by ReproSeq Low-pass $v 1.0$ tool of Ion Reporter version 5.0 suite. c.707-1G>A mutation was analyzed by minisequencing and using DXS1275, DXS8031, and DXS1690 y DXS8107 markers by fragment analysis and amelogenin (AME$L Y$ ) gene for detection of $Y$ chromosome by quantitative PCR.

\section{RESULTS}

\section{Patients}

This family is affected by X-linked HED with three affected members, two deceased brothers (Fig. 1, individuals III-5 and III-8) and one nephew (Fig. 1, individual IV-8) of the proband. The proband (individual III-3, marked by an arrow in Fig. 1) is a 25-year-old woman who has two missing teeth (upper right and left lateral incisors), with no other features of HED. Her partner is a 26-year-old healthy man. The couple was referred to our infertility clinic for their interest in PGT for X-linked HED before attempting to conceive.

The patient is a carrier of a novel pathogenic genetic variant NM_001399.4:c.707-1G>A (rs886039466) in EDA gene (OMIM 300451). This variant was included in ClinVar database (SCV000322044.6) by GeneDx laboratory, which originally performed molecular testing and classified it as pathogenic. It is proposed that the mutation removes the canonical splice acceptor site in intron 4 and causes abnormal gene splicing. The rs886039466 variant is not reported in the 123,136 exomes, and 15,496 genomes included in the gnomAD database or the 62,784 individuals of TOPMed database, supporting that this is a rare variant even in Latino population.

\section{PGT cycles}

In the first PGT cycle, 11 oocytes were retrieved, of and seven were in metaphase II. Five blastocysts developed after intracytoplasmic sperm injection (ICSI); three blastocysts of excellent quality were biopsied with the following results: Two non-carrier, non-aneuploid $X Y$ embryos, and one $X X$ carrier, $-10 q$ abnormal embryo. The two non-carrier $X Y$ blastocysts were transferred with a negative $\beta$-gonadotropin result 2 weeks after transfer.

For a second PGT cycle, we decided to change the stimulation approach and accumulate zygotes before the blastocyst biopsy to have a better chance of pregnancy and decrease the cost of genetic testing. Three ovarian stimulations were performed obtaining six metaphase II oocytes and six vitrified zygotes in the first stimulation, and seven metaphase II oocytes and six vitrified zygotes in the second. In the third and last stimulation, 12 metaphase II oocytes were retrieved, and nine zygotes were obtained. The 12 zygotes from two previous stimulations were thawed, and all embryos were cultured to blastocyst stage having a total of 10 blastocysts biopsied for the second PGT cycle. The results of combined genetic diagnosis of the second PGT are presented in table 1. 
Table 1. Results of combined genetic analysis for EDA c.707-1G>A mutation and aneuploidy in the second PGT cycle.

\begin{tabular}{|c|c|c|c|c|c|c|c|}
\hline Embryo & Origin & $\begin{array}{l}\text { Mutation and } \\
\text { maternal haplotype }\end{array}$ & $\begin{array}{l}\text { Aneuploidy } \\
\text { screening }\end{array}$ & Sex & $\begin{array}{l}\text { Combined } \\
\text { diagnosis }\end{array}$ & \multicolumn{2}{|c|}{$\begin{array}{l}\text { Recommended } \\
\text { for transfer }\end{array}$} \\
\hline 1 & Fresh oocyte & Negative & No imbalance & $X X$ & $\begin{array}{l}\text { XX, not a carrier, } \\
\text { no aneuploidy }\end{array}$ & Yes & $\frac{\text { है }}{\frac{\delta}{L}}$ \\
\hline 2 & Fresh oocyte & Positive & No imbalance & $X X$ & $\begin{array}{l}\text { XX, carrier, } \\
\text { no aneuploidy }\end{array}$ & \multicolumn{2}{|c|}{$\begin{array}{l}\text { Counseling for } \\
\text { female carrier }\end{array}$} \\
\hline 3 & Fresh oocyte & Positive & No imbalance & $X X$ & $\begin{array}{l}\text { XX, carrier, } \\
\text { no aneuploidy }\end{array}$ & \multicolumn{2}{|c|}{$\begin{array}{l}\text { Counseling for } \\
\text { female carrier }\end{array}$} \\
\hline 4 & Fresh oocyte & Negative & No imbalance & $X Y$ & $\begin{array}{l}X Y, \text { not a carrier, } \\
\text { no aneuploidy }\end{array}$ & Yes & $\frac{0}{\frac{0}{2}}$ \\
\hline 5 & Fresh oocyte & Negative & $+16 p,+21$ & $X Y$ & $\begin{array}{l}\mathrm{XY} \text {, not a carrier, } \\
\text { aneuploidy }\end{array}$ & No & $\frac{1}{+}$ \\
\hline 6 & Fresh oocyte & Negative & $-9 q$ & $X X$ & $\begin{array}{l}\text { XX, not a carrier, } \\
\text { aneuploidy }\end{array}$ & No & $\frac{\bar{o}}{\sqrt[n]{n}}$ \\
\hline 7 & Vitrified embryo & Positive & -16 & $X X$ & $\begin{array}{l}\text { XX, carrier, } \\
\text { aneuploidy }\end{array}$ & No & $\stackrel{\bar{\Xi}}{\varrho}$ \\
\hline 8 & Vitrified embryo & Negative & $+12,+16 p$ & $X X$ & $\begin{array}{l}\text { XX, not a carrier, } \\
\text { aneuploidy }\end{array}$ & No & $\frac{ \pm}{3}$ \\
\hline 9 & Vitrified embryo & Positive & $+5 q,+7,-16,+21$ & $X X$ & $\begin{array}{l}\text { XX, carrier, } \\
\text { aneuploidy }\end{array}$ & No & $\frac{\bar{x}}{\bar{c}}$ \\
\hline 10 & Vitrified embryo & Negative & No imbalance & $X Y$ & $\begin{array}{l}\text { XY, not a carrier, } \\
\text { no aneuploidy }\end{array}$ & Yes & $\stackrel{5}{\ddagger}$ \\
\hline
\end{tabular}

PGT: preimplantation genetic testing.

Embryo number 1 in table 1 was transferred; this was an $X X$, non-carrier, and euploid embryo. At 14 days after transfer, the level of hCG was $740 \mathrm{IU} / \mathrm{mL}$. Vaginal ultrasound confirmed a gestational sac with heartbeat at 35 days. The couple was not interested in performing prenatal genetic testing; however, a structural ultrasound at 12.5 and 22 weeks was performed to rule out malformations. An apparently healthy girl was delivered by cesarean section at 35.4 weeks, indicated because of premature labor. She had 36 weeks estimated by Capurro's gestational score, weight of $2580 \mathrm{~g}$ and height of $47 \mathrm{~cm}$ with an Apgar score of $8 / 9$. Karyotype in blood was $46, X X$ [30], confirming the results of PGT-A; the mutation in the EDA gene has not been tested in the baby.

Other two euploid, non-carrier blastocysts (Table 1) are available in case the couple is interested in a second child.

\section{DISCUSSION}

The mutation spectrum of $X$-linked HED has been reported in different cohorts of Mexican mestizo patients $s^{5,6}$, but the variant found in the reported family is not included in those cohorts. Since several Mexican families have been reported with $X$-linked HED, clearly prenatal diagnosis of pathogenic mutations is a necessary tool to support these families in Mexico. Our report introduces PGT as an alternative for families looking for prenatal diagnosis of $X$-linked HED using next-generation sequencing, and it can be considered as a model for other $X$-linked recessive disorders such as hemophilia A, Menkes disease, or $X$ linked adrenoleukodystrophy.

In Mexico, assisted reproduction is a well-developed branch of gynecology with more than 40 clinics accredited by the Latin-American Network of Assisted Reproduction (REDLARA). Delivery rates per cycle in REDLARA are $25.05 \%$ for ICSI and $27.41 \%$ for IVF, respectively ${ }^{7}$, which are very similar to results in the United States and the best centers in Europe. However, PGT in all of Latin America is importantly limited compared to Europe and the US, with 2119 PGT cycles and only 95 deliveries, with a delivery rate per initiated fresh cycle of $4.7 \%$ and $36.40 \%$ delivery rate per embryo transfer in $2014^{7}$. Unfortunately, the 
REDLARA data on $\mathrm{PGT}^{7}$ do not differentiate between PGT-A and PGT-M cycles to better estimate how often PGT-M is used in Latin-American countries to prevent Mendelian disorders.

On the other hand, Mexico is slowly incorporating molecular testing for mutations in patients affected by genetic disorders, including those requiring assisted reproduction as treatment for infertility ${ }^{8}$ and using new techniques for molecular diagnosis based on parallel massive sequencing, such as exome sequencing 9 . Soon, when it becomes common that patients undergo testing to identify the mutations producing their genetic disorders, they will request prenatal and preimplantation diagnosis more often as part of their reproductive decisions.

A growing experience in the United States and Europe is showing that a single blastocyst biopsy can be used for diagnosis of monogenic disorders or chromosome translocations and aneuploidy screening 2,10 . The rationale of testing for aneuploidy in PGT-M cycles is the high prevalence of aneuploidy in embryos from non-infertile couples; for example, only $25 \%$ of blastocysts have been reported as euploid and not affected by mutations in a cohort of young women undergoing $\mathrm{PGT}^{11}$. Other authors reported similar numbers ${ }^{10}$, strongly supporting the importance of concurrent PGT-M and PGT-A testing. Other new PGT techniques, such as karyomapping, offer the potential for diagnosing recessive monogenic disorders and aneuploidies at the same time ${ }^{12}$ without the need of setting-up a genetic diagnosis for each family, as was done in the case here reported.

Rare diseases are defined as those occurring with a frequency of $<1: 2000$ individuals. They are mainly genetic ( $80 \%)$, and it is estimated that 300 million individuals worldwide are affected by a rare disease. Therefore, there is a general necessity among physicians practicing internal and family medicine to diagnose, personalize follow-up, treat, and importantly, prevent these disorders.

In conclusion, we report the first experience using a combined diagnosis of a monogenic disorder and aneuploidies in Mexico. This approach should be considered as a new tool for families affected by monogenic genetic disorders, including $X$-linked, trying to prevent the transmission of disease. We foresee that in the era of personalized medicine, assisted reproduction will have a primary role in the prevention of diseases in families affected by rare genetic disorders.

\section{ACKNOWLEDGMENTS}

The authors thank the family who kindly accepted to share their experience with HED and allowed us to report the results of the PGT cycles in this manuscript.

\section{REFERENCES}

1. Wright JT, Grange DK, Fete M, et al. Hypohidrotic ectodermal dysplasia. 1993.

2. Zimmerman RS, Jalas C, Tao X, et al. Development and validation of concurrent preimplantation genetic diagnosis for single gene disorders and comprehensive chromosomal aneuploidy screening without whole genome amplification. Fertil Steril. 2016;105:286-94.

3. Martínez-Robles IM, González-Ortega C, Saavedra-Campos P, et al. Administración de hormona luteinizante recombinante ( $\mathrm{LHr}$ ) como protocolo de estimulación ovárica controlada en FIV-ICSI. Ginecol Obstet Mex. 2016;84:630-8.

4. González-Ortega C, Cancino-Villarreal P, Pérez-Torres A, et al Inyección intracitoplásmica de espermatozoides morfológicamente seleccionados (IMSI) vs inyección intracitoplásmica de espermatozoides (ICSI) en pacientes con falla repetida a ICSI. Ginecol Obstet Mex. 2010;78:652-9.

5. Salas-Alanis JC, Wozniak E, Mein CA, et al. Mutations in EDA and EDAR genes in a large Mexican Hispanic cohort with hypohidrotic ectodermal dysplasia. Ann Dermatol. 2015;27:474-7.

6. Monroy-Jaramillo N, Abad-Flores JD, García-Delgado C, et al. Mutational spectrum of EDA and EDAR genes in a cohort of Mexican Mestizo patients with hypohidrotic ectodermal dysplasia. J Eur Acad Dermatol Venereol. 2017;31:e321-4.

7. Zegers-Hochschild F, Schwarze JE, Crosby J, Musri C, Urbina MT; Latin American Network of Assisted Reproduction (REDLARA). Assisted reproduction techniques in Latin America: the Latin American Registry, 2014. Reprod Biomed Online. 2017;35:287-95.

8. Piña-Aguilar RE, Regalado-Hernández MÁ, Moreno-García JD, et al. A rapidly progressive defective spermatogenesis in a Mexican family affected by spino-bulbar muscular atrophy. Syst Biol Reprod Med. 2016;62:146-51.

9. Chacon-Camacho OF, Jitskii S, Buentello-Volante B, QuevedoMartinez J, Zenteno JC. Exome sequencing identifies RDH12 compound heterozygous mutations in a family with severe retinitis pigmentosa. Gene. 2013;528:178-82.

10. Minasi MG, Fiorentino F, Ruberti A, et al. Genetic diseases and aneuploidies can be detected with a single blastocyst biopsy: a successful clinical approach. Hum Reprod. 2017;32:1770-7.

11. Goldman KN, Nazem T, Berkeley A, Palter S, Grifo JA. Preimplantation genetic diagnosis (PGD) for monogenic disorders: the value of concurrent aneuploidy screening. J Genet Couns. 2016; 25:1327-37

12. Natesan SA, Bladon AJ, Coskun S, et al. Genome-wide karyomapping accurately identifies the inheritance of single-gene defects in human preimplantation embryos in vitro. Genet Med. 2014;16:838-45 